\title{
Magnetic beads retention device for sandwich immunoassay: comparison of off-chip and on-chip antibody incubation
}

\author{
F. Lacharme $\cdot$ C. Vandevyver $\cdot$ M. A. M. Gijs
}

Received: 24 October 2008/ Accepted: 15 December 2008/Published online: 14 March 2009

(C) Springer-Verlag 2009

\begin{abstract}
We use magnetic microbeads, which are magnetically self-assembled in chains in a microfluidic chip, as reaction substrates to implement two different sandwich immunoassay protocols for the detection of mouse monoclonal target antibodies. The magnetic chains form when the chip is placed in a magnetic field, and are geometrically trapped and accurately positioned in a microchannel with periodically enlarged cross-sections. In the first immunoassay protocol, capture and target antibodies are incubated offchip, while exposure to the detection antibody is performed on-chip. In the second protocol, the complete immunoassay is fully executed on-chip. In the 'off-chip incubation-onchip detection' protocol, antibodies can be detected down to a concentration of $50 \mathrm{ng} / \mathrm{mL}$ in a total assay time of $120 \mathrm{~min}$, while consuming $1.5 \mathrm{~mL}$ of target antibody solution. Using the full on-chip protocol, our system is able to detect target antibodies in the range of a few $\mathrm{ng} / \mathrm{mL}$ in $30 \mathrm{~min}$, using only a few tens of nanoliters of target antibody solution and reagents. The 'off-chip incubation-on-chip detection' protocol is also applied for dosing antibodies obtained from the supernatant of a cell culture medium.
\end{abstract}

Keywords Microfluidics - Immunoassay · ELISA · Antibody · Magnetism · Magnetic microbeads . Self-assembly $\cdot$ Microchip $\cdot$ Nanoparticles

F. Lacharme · M. A. M. Gijs $(\bowtie)$

Laboratory of Microsystems, Ecole Polytechnique Fédérale

de Lausanne (EPFL), Batiment BM, Station 17,

1015 Lausanne, Switzerland

e-mail: martin.gijs@epfl.ch

C. Vandevyver

Research Commission EPFL-SNF, Ecole Polytechnique

Fédérale de Lausanne (EPFL), 1015 Lausanne, Switzerland

\section{Introduction}

The miniaturization of analytical chemistry systems has become a major challenge in the research community since the introduction of the micro total analysis system ( $\mu$-TAS) in 1990 (Manz et al. 1990). Immunoassay analyses (for example the Enzyme-Linked Immunosorbent Assay (ELISA), an analytical technique widely used in clinical diagnostics, cancer research, and food safety or environmental analysis) use the sensitivity and specificity of highly stable interactions between antigens $(\mathrm{Ag})$ and their specific antibodies $(\mathrm{Ab})$. An immunoassay analysis is conventionally performed in microtiter plates, and consumes typically about hundreds of $\mu \mathrm{L}$ of sample and reagent solutions, for an assay time of hours due to mass-transport limitations. In microfluidics, the small reaction volume and microchannel dimensions permit a strong analysis time reduction, while consuming minute quantities of samples and reagents (typically a few $\mu \mathrm{L}$ ). However, microfluidic integrated systems developed for immunoassays demand additional steps for a perfect control of microchannel surface chemistry, to allow an efficient and reproducible $\mathrm{Ab}$ immobilization. This drawback can be circumvented by using functionalized microbeads in a fluidic chip. Microbeads are available at different sizes, present the advantage of a large surface-to-volume ratio, and offer a strong flexibility due to a wide range of available surface chemistries. Their use as substrate for microfluidic immunoassays was investigated by elaborating microbead retention strategies at desired positions within a microchannel, using for example a microdam (Sato et al. 2001) or micropillar cage (Andersson et al. 2000). When microbeads incorporate a magnetic core, they can be easily manipulated using external magnetic fields, explaining why they are increasingly used as a solid phase for Ab capture and as carriers of 
captured Ag (Gijs 2004). Their retention at the microchannel wall was realized using macroscopic permanent magnets (Hayes et al. 2001), current-activated coils (Choi et al. 2002) and wires (Siegel et al. 2006), or magnetic structures (Deng et al. 2002; Lund-Olesen et al. 2007) incorporated on the microchip. Mobile permanent magnets were also used for magnetic microbeads' manipulation in a microfluidic chip incorporating a dual channel network, 460 $\mathrm{nL}$ reaction chambers and valve-activated fluidic bridges (Herrmann et al. 2006, 2007). Recently, the manipulation and retention of magnetic microbeads over the complete cross-section of a microchannel was proposed using a focused magnetic field (Rida and Gijs 2004; Slovakova et al. 2005; Smistrup et al. 2005; Bronzeau and Pamme 2008; Peyman et al. 2008), or by trapping self-assembled magnetic chains geometrically in a periodically structured microchannel (Lacharme et al. 2008a, b). In these systems, the intense liquid-magnetic microbead interactions lead to a highly efficient active mixing or target analyte capture.

In this paper, we present and compare two protocols to perform on-chip immunoassays using our magnetic microbead retention device. The magnetic microbeads are incubated with capture $\mathrm{Ab}(\mathrm{c}-\mathrm{Ab})$ and target monoclonal $\mathrm{Ab}(\mathrm{t}-\mathrm{mAb})$ either off-chip or directly on-chip (t-mAb is used as target antigen in our sandwich immunoassay), while exposure to the detection $\mathrm{Ab}$ (d-Ab) is always performed on-chip. The off-chip incubation-on-chip detection protocol extends the use of our chip to magnetic microbead-based analytical methods involving classical bench-top $\mathrm{Ab}$ analysis procedures. A controlled amount of magnetic microbeads is self-assembled in magnetic chains that are accurately retained in the large sections of a periodically enlarged microchannel, by simply placing the chip in an external homogeneous magnetic field. The self-assembled magnetic chains strongly interact with analytes and $\mathrm{d}-\mathrm{Ab}$ from the perfusing microfluidic flows. We compare the performance of both immunoassay protocols and, as additional application example, demonstrate the dosing of $\mathrm{t}-\mathrm{mAb}$ produced by a hybridoma cell culture.

\section{Microsystem design and fabrication}

The schematic layout of the microchip showing the periodical enlargement of the microchannel is presented in Fig. 1a. It consists of a $20-\mu \mathrm{m}$-wide microchannel with periodical sections of $40 \mu \mathrm{m}$ (of $5 \mu \mathrm{m}$ length) separated by $10 \mu \mathrm{m}$. 130 sections of this type are realized over a total distance of $2 \mathrm{~mm}$. The microchips are microfabricated using a dry-etching technology (Pavius 2004), to obtain a vertical microchannel wall profile. Briefly, a $2-\mu \mathrm{m}$-thick layer of amorphous silicon (a-Si) is deposited on a 4-inch Pyrex wafer, following by spinning, exposure, and development of a positive photoresist
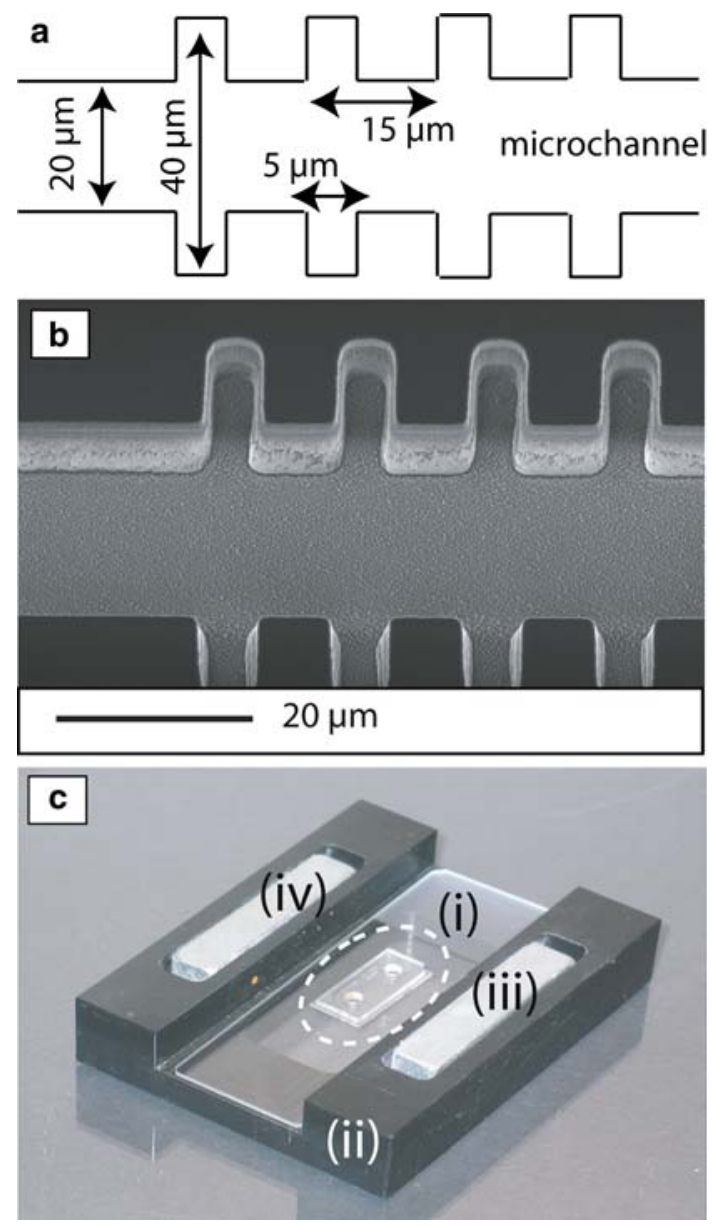

Fig. 1 a Schematic layout of the $20-\mu \mathrm{m}$-wide microchannel showing the periodically enlarged sections of $40 \mu \mathrm{m}$. b SEM picture of the microchannel showing the periodically varying channel width. c Picture of the microfluidic chip in its socket. The chip consists of a 8 - $\mu$ m-deep plasma-etched channel in a Pyrex substrate that is sealed with a $2.5 \mathrm{~mm}$ thick Polydimethylsiloxane (PDMS) cover (i). The latter is pinched with two access holes and placed on the chip socket (ii) that contains two permanent magnets (iii, iv) generating a homogeneous magnetic field

using a standard photolithography process. The a-Si is dry etched using a deep reactive ion-etching (DRIE) technique, and the remaining photoresist is stripped. Then, the Pyrex wafer is DRIE etched to a depth of $8 \mu \mathrm{m}$, followed by removal of the a-Si protective mask in a $40 \% \mathrm{KOH}$ bath, heated at $60^{\circ} \mathrm{C}$ during $\sim 1 \mathrm{~h}$. Finally, the wafer is cut in individual microchips. Figure $1 \mathrm{~b}$ shows a scanning electron microscopy (SEM) image of the microchannel. Finally, the microchip is loosely covered with a $2 \mathrm{~mm}$ thick polydimethylsiloxane (PDMS) cover, which is pinched with two access holes forming two reservoirs (reservoir A and B). Figure 1c shows a picture of the microchip (i) on its holder used during the experiments, where it is placed at the center of a poly-oxymethylene support (ii), and flanked by two permanent magnets (iii and iv) for the generation of the permanent homogeneous magnetic field (Rida et al. 2003). 


\section{Magnetic bead retention principle}

A solution of $500 \mathrm{~nm}$ diameter magnetic microbeads is introduced into the microchannel. When a homogeneous magnetic field is applied perpendicularly to the microchannel axis, the magnetic microbeads self-assemble in magnetic chains (Doyle et al. 2002) in the microchannel's large cross-sections, thereby decreasing their total magnetic dipolar energy. Figure 2a is a schematic drawing of the system where a flow is applied through a magnetic chain, exerting a drag force $F_{\text {drag }}$ which displaces the central part of the chain over a distance $X$. The saturation magnetization of the microbeads is $M_{\text {sat }}=8 \times 10^{4} \mathrm{~A} / \mathrm{m}$. Then, the maximal magnetic moment of one bead $m_{\text {bead }}=(4 / 3) \pi R^{3} M_{\text {sat }}=5.23 \times 10^{-15} \mathrm{Am}^{2}$, where $R$ is the bead radius $(250 \mathrm{~nm})$. We measure with a miniaturized Hall probe a magnetic induction of $120 \mathrm{mT}$ originating from the two permanent magnets at the center of the chip holder, where the microchannel is inserted during experiments. This magnetic induction is large enough to saturate the magnetic moment of the microbeads. Assuming that all the magnetic microbeads have reached their magnetic saturation (due to the permanent magnetic field) and are individual dipoles oriented in the external magnetic field

a
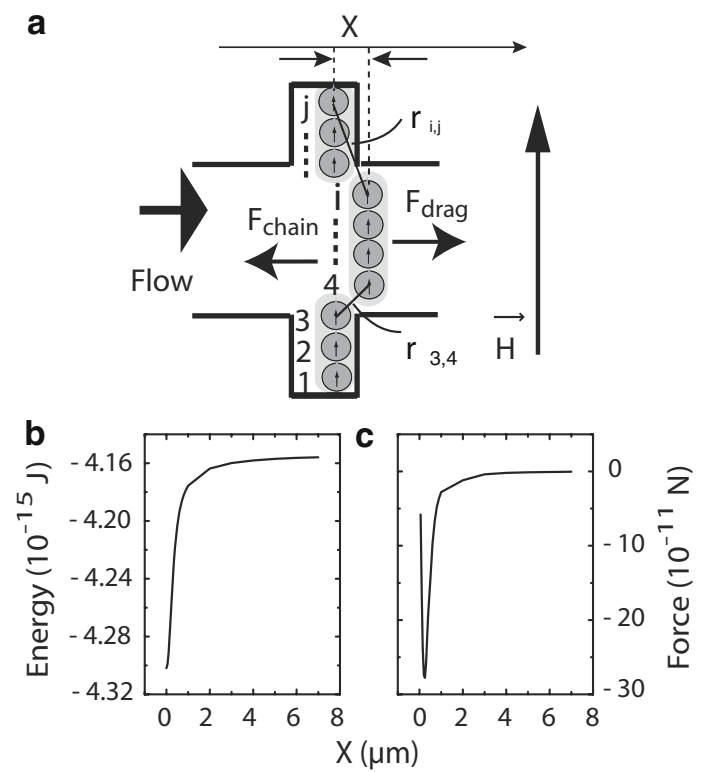

Fig. 2 a Schematic layout of the microchannel with periodically varying channel width. In a magnetic field $\mathbf{H}$, the magnetic microbeads self-assemble into chains that stay trapped in the large cross-sections. When a fluid flow is applied, the viscous drag force displaces the center part of the microbead chains. $r_{i, j}$ is the distance between the nanoparticles with index $i$ and $j$, respectively. b Magnetic dipolar energy $E_{\text {chain }}$ of a chain consisting of 80 magnetic microbeads as a function of the displacement $X$ induced by the flow. c Magnetic retention force $F_{\text {chain }}$ of a chain consisting of 80 microbeads as a function of $X$ direction, the magnetic dipole energy of a pearl-like chain of $n$ microbeads is given by (Helseth 2005)

$E_{\text {chain }}=\sum_{i=0}^{i=n} \sum_{j>i}^{n} \frac{\mu_{0}}{4 \pi} \frac{1}{r_{i j}^{3}} m_{\text {bead }}^{2} \times\left(1-\cos ^{2} \Theta_{i j}\right)$

where $\mu_{0}$ is the magnetic permeability of vacuum, $r_{i j}$ the distance between the microbeads with index $i$ and $j$, and $\Theta_{i j}$ the angle between the external magnetic field direction and the center-to-center vector of microbeads with index $i$ and $j$, respectively. When exposed to the flow, the viscous drag force acting on the central part of 80- microbead pearl-like magnetic chain is approximated by the Stokes drag force:

$F_{\text {drag }}=6 C \pi \eta R v$

where $C$ is the number of magnetic microbeads in the center part of the chain (40 for a 80-magetic microbead pearl-like chain trapped in a $40 \mu \mathrm{m}$ enlarged microchannel section), $\eta$ the liquid viscosity and $v$ the velocity of the liquid with respect to the magnetic microbeads. Figure $2 b$ presents the magnetic dipolar energy $E_{\text {chain }}$ for a chain of 80 microbeads calculated using Eq. 1, as a function of $X$. The magnetic energy of the chain increases with $X$, as the individual microbead center-to-center distances increase. This energy variation leads to a magnetic dipolar force given by

$F_{\text {chain }}=-\frac{\partial E_{\text {chain }}}{\partial X}$.

Figure $2 \mathrm{c}$ presents the variation of the magnetic dipolar force $F_{\text {chain }}$ as a function of $X$ calculated using Eq. 3. A maximum force of $\sim 270 \mathrm{pN}$ is calculated for a distance $X=250 \mathrm{~nm}$. When the drag force is superior to the magnetic force $\left(F_{\mathrm{drag}}>F_{\text {chain }}\right)$, the central part of the magnetic chain is no longer retained and the chain breaks in three parts. Equalizing $F_{\text {drag }}$ and $F_{\text {chain }}$ lead to a critical mean fluid velocity for chain rupture of $v_{c}=1.4 \mathrm{~mm} / \mathrm{s}$. During our experiment, we typically use a maximum flow velocity at the microchannel center of $160 \mu \mathrm{m} / \mathrm{s}$ (i.e., a flow rate of $0.034 \mathrm{~nL} / \mathrm{s}$ ). Then, no chain breaking is observed during experiments, and the self-assembled magnetic chains stay retained and are accurately positioned at the entire crosssection of microchannel.

\section{Experimental}

All $\mathrm{c}-\mathrm{Ab}$ and $\mathrm{d}-\mathrm{Ab}$ solutions are prepared by diluting the desired amount of $\mathrm{Ab}$ in phosphate buffer saline-bovine serum albumine (PBS-BSA) solution. (PBS-BSA) solution is prepared by diluting $1 \%(\mathrm{w} / \mathrm{w})$ BSA (obtained from Sigma-Aldrich, product no. A7030) in PBS (obtained from Sigma-Aldrich, product no. P5493) solution (pH 7.4). In this study, all Ab used are immunoglobulin $\mathrm{G}(\mathrm{IgG})$. The 
c-Ab molecules used for both on- and off-chip immunoassay are rabbit biotinylated anti-mouse $\operatorname{IgG}$ (obtained from Dako, product no. E0354). The direct detection Ab (dd-Ab) molecules used for performing a controlling direct immunoassay are $\mathrm{Cy} 3$-conjugated polyclonal goat antirabbit IgG (obtained from Bioconcept, product no. 601104-122). The sandwich detection $\mathrm{Ab}$ (sd-Ab) molecules used for the sandwich immunoassay are $\mathrm{Cy} 3$-conjugated polyclonal goat anti-mouse IgG (obtained from BioConcept, product no. 610-104-121). The t-mAb molecules used for the sandwich immunoassay are normal mouse $\operatorname{IgG}$ (obtained from Invitrogen, product no. 10400C). The t-mAb solutions are prepared by diluting $39 \mu \mathrm{g} / \mathrm{mL}$ initial concentrated solution in cell culture medium (obtained from Invitrogen) supplemented with 5\% fetal calf serum (FCS, obtained from Invitrogen). Streptavidin coated magnetic microbeads (MasterBeads Streptavidin obtained from Ademtech, product no. 03153) are used as magnetic microbeads for both the on- and off-chip immunoassay. Immobilization Buffer (obtained from Ademtech, product number no. 10301) is used as washing buffer (WB), according to the supplier's recommendations. For both protocols, before introducing magnetic microbeads in the microchannel, we first pour a surface-blocking solution through the microchip to avoid aspecific $\mathrm{Ab}$ adsorption on the glass channel wall and on the PDMS cover. A volume of $15 \mu \mathrm{L}$ of PBS-BSA solution is introduced in one reservoir (reservoir A), after which the solution fills the entire microchannel by capillary forces within a few seconds. Then, the second reservoir (reservoir B) is also filled with $15 \mu \mathrm{L}$ of PBS-BSA solution. After $5 \mathrm{~min}$, both reservoirs are emptied by withdrawing solutions using a micropipette, and rinsed with $15 \mu \mathrm{L}$ of WB.
The 'off-chip incubation-on-chip detection' protocol is illustrated in Fig. 3. In a first step, $10 \mu \mathrm{L}$ of magnetic microbead solution is resuspended in $10 \mu \mathrm{L}$ of $\mathrm{WB}$, to avoid magnetic beads' clustering or reaction vial surface adsorption before $\mathrm{c}-\mathrm{Ab}$ incubation. The magnetic microbeads are then incubated for $30 \mathrm{~min}$ under gentle agitation with $166 \mu \mathrm{L}$ of c-Ab diluted at $455 \mathrm{ng} / \mathrm{mL}$ (Fig. 3i). Using this $\mathrm{c}-\mathrm{Ab}$ solution concentration, the size of magnetic microbead agglomerates formed because of the link between the surface streptavidin molecules and the $\mathrm{c}-\mathrm{Ab}$ is limited, allowing the functionalized magnetic microbeads to be easily introduced in microchannel. Then, the magnetic microbeads are washed with $500 \mu \mathrm{L}$ of $\mathrm{WB}$ and resuspended in $10 \mu \mathrm{L}$ of WB. In a second step, the microbeads are incubated with $500 \mu \mathrm{L}$ of $\mathrm{t}-\mathrm{mAb}$ solution (Fig. 3ii) at different concentrations (from 50 to $200 \mathrm{ng} / \mathrm{mL}$ ) for 60 min under gentle agitation. Then, the microbeads are washed with $500 \mu \mathrm{L}$ of WB and resuspended in $10 \mu \mathrm{L}$ of WB, avoiding further magnetic bead adsorption to the microchannel surface. The final magnetic microbead concentration is of $10 \mathrm{mg} / \mathrm{mL}$ (Fig. 3iii). A volume of $2 \mu \mathrm{L}$ of the magnetic microbead solution is introduced in reservoir A and flushed into the microchannel by applying a short over-pressure in the reservoir. Then, the microchip is placed on its holder supporting the permanent magnets. All magnetic chains are self-assembled after $\sim 10 \mathrm{~s}$ (Fig. 3iv). The magnetic microbead solution remaining in reservoir A is withdrawn from the microchip with a micropipette, and reservoir $\mathrm{A}$ is filled with $4 \mu \mathrm{L}$ of PBS-BSA solution. To execute the on-chip detection, we fill reservoir $\mathrm{B}$ with $15 \mu \mathrm{L}$ of sd-Ab solution at $5 \mu \mathrm{g} / \mathrm{mL}$ (Fig. $3 \mathrm{v}$ ). The liquid volume difference between reservoirs $\mathrm{A}$ and $\mathrm{B}$ provides the pressure to generate a liquid flow velocity of $160 \mu \mathrm{m} / \mathrm{s}$ at

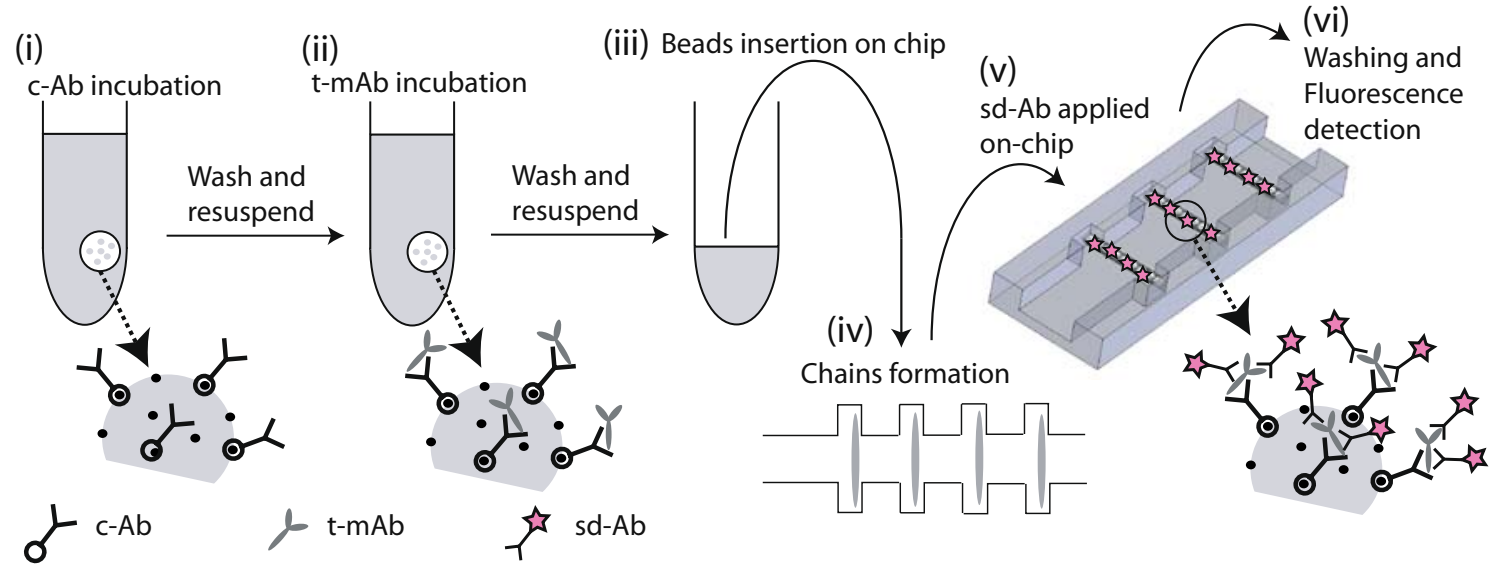

Fig. 3 Illustration of the 'off-chip incubation-on-chip detection' protocol. $i$ The magnetic microbead solution is incubated with capture antibodies $(c-A b)$. ii The microbeads are incubated with target monoclonal antibodies $(t-m A b)$ solution at different concentrations under gentle agitation. iii The magnetic microbeads are introduced in the microchip, where (iv) the magnetic chains selfassemble. $v$ Flow of the sandwich detection antibodies $(s d-A b)$ solution through the magnetic chains. vi After a washing step, the fluorescence of the chains can be detected 
Table 1 Summary of the experimental parameters used for the three experimental protocols

\begin{tabular}{|c|c|c|c|c|c|}
\hline Experimental step & $\begin{array}{l}\text { Total number } \\
\text { of beads }\end{array}$ & $\begin{array}{l}\text { Ab solution } \\
\text { volume }(\mathrm{nL})\end{array}$ & $\begin{array}{l}\text { Ab solution } \\
\text { concentration } \\
(\mathrm{ng} / \mathrm{mL})\end{array}$ & $\begin{array}{l}\text { Total number of } \mathrm{Ab} \\
\text { molecules }\end{array}$ & $\begin{array}{l}\text { Average number of } \mathrm{Ab} \\
\text { molecules per bead }\end{array}$ \\
\hline \multicolumn{6}{|c|}{ Off-chip incubation-on-chip detection } \\
\hline Incubation of c-Ab (off-chip) & $7.63 \times 10^{8}$ & $166 \times 10^{3}$ & 455 & $3.02 \times 10^{11}$ & 396 \\
\hline Incubation of t-Ag (off-chip) & $7.63 \times 10^{8}$ & $500 \times 10^{3}$ & From 50 to 200 & From $1 \times 10^{11}$ to $6 \times 10^{11}$ & From 131 to 786 \\
\hline Application of sd-Ab (on-chip) & 29,000 & 15.3 & 5,000 & $3.24 \times 10^{8}$ & 11,066 \\
\hline \multicolumn{6}{|l|}{ Full on-chip direct immunoassay } \\
\hline Application of $\mathrm{c}-\mathrm{Ab}$ & 29,000 & 6.12 & 5,000 & $1.22 \times 10^{8}$ & 4,167 \\
\hline Application of dd-Ab & 29,000 & 10.2 & 5,000 & $2.04 \times 10^{8}$ & 6,967 \\
\hline \multicolumn{6}{|l|}{ Full on-chip sandwich immunoassay } \\
\hline Application of $\mathrm{c}-\mathrm{Ab}$ & 29,000 & 6.12 & 5,000 & $1.22 \times 10^{8}$ & 4,167 \\
\hline Application of $\mathrm{t}-\mathrm{mAb}$ & 29,000 & 30.6 & From 1 to 200 & From $1.22 \times 10^{4}$ to $2.45 \times 10^{6}$ & From 4 to 836 \\
\hline Application of $\mathrm{sd}-\mathrm{Ab}$ & 29,000 & 10.2 & 5,000 & $3.24 \times 10^{8}$ & 11,066 \\
\hline
\end{tabular}

the microchannel center (Shui et al. 2007), without the need of external complex tubing or pumping system. The $\mathrm{sd}-\mathrm{Ab}$ solution is flown through the microchannel for $7.5 \mathrm{~min}$, to ensure an excess amount of $\mathrm{d}-\mathrm{Ab}$ with respect to the $\mathrm{t}-\mathrm{mAb}$. Then, reservoir $\mathrm{B}$ is rapidly rinsed (with WB), before application during $3 \mathrm{~min}$ of WB through the microchannel by filling reservoir $B$ with $15 \mu \mathrm{L}$ of WB solution. Finally, reservoir B is filled with $15 \mu \mathrm{L}$ of PBSBSA solution during fluorescence detection (Fig. 3vi).

The full on-chip immunoassay protocol has been previously described in detail (Lacharme et al. 2008a, b). Briefly, $2 \mu \mathrm{L}$ of $10 \mathrm{mg} / \mathrm{mL}$ magnetic microbead solution (resuspended in WB to avoid magnetic beads adsorption to the microchannel wall surface) is introduced in reservoir A (before $\mathrm{c}-\mathrm{Ab}$ coating), and flushed into the microchannel by applying a short over-pressure in reservoir $\mathrm{A}$. The microchip is placed on its holder containing the permanent magnets and chains form. Excess of magnetic microbead solution is withdrawn from reservoir A using a micropipette and replaced by $4 \mu \mathrm{L}$ of PBS-BSA solution. All other fluid operations are performed by filling and withdrawing $15 \mu \mathrm{L}$ of different solutions from reservoir B. The on-chip direct control immunoassay is performed by subsequently letting flow through the microchannel (from reservoir B to reservoir A) solutions of (1) c-Ab at $5 \mu \mathrm{g} / \mathrm{mL}$ for $3 \mathrm{~min}$ and (2) dd-Ab at $5 \mu \mathrm{g} / \mathrm{mL}$ for $5 \mathrm{~min}$. The sandwich immunoassay is performed by subsequently letting flow through the microchannel (from reservoir B to reservoir A) solutions of (1) c-Ab at $5 \mu \mathrm{g} / \mathrm{mL}$ (in PBS only solution) for $3 \mathrm{~min}$, (2) $\mathrm{t}-\mathrm{mAb}$ solutions at different concentrations (from 1 to $200 \mathrm{ng} / \mathrm{mL}$ ) for $15 \mathrm{~min}$ and (3) $\mathrm{sd}-\mathrm{Ab}$ at $5 \mu \mathrm{g} / \mathrm{mL}$ for 5 min. All steps (during both the direct and sandwich immunoassay) are separated by a fast-rinsing $(\sim 10 \mathrm{~s})$ and 3 min washing step using $15 \mu \mathrm{L}$ of WB solution introduced in reservoir B. Finally, the reservoir B is filled with $15 \mu \mathrm{L}$ of PBS-BSA solution during fluorescence detection. Table 1 summarizes the experimental parameters (total number of magnetic microbeads used, Ab solution volume and $\mathrm{Ab}$ concentration, total number of $\mathrm{Ab}$ molecules and average number of $\mathrm{Ab}$ molecules per bead) used for the three experimental protocols.

For visualization, image acquisition, and fluorescence detection, we use a CCD camera ORCA-C4742-95ER (Hamamatsu) mounted on an inverted microscope Axiovert S100 (Zeiss), with a $100 \times$ objective (LD EC EpiplanNeofluar, numerical aperture 0.75 ), a $40 \times$ objective (LD Achroplan, numerical aperture 0.5 ) and a $10 \times$ objective (Epiplan, numerical aperture 0.2). The microscope is equipped with a Mercury short arc lamp HBO (Osram) and the appropriate filter set for Cy3 dye (filter set no. 20, Zeiss). The excitation, dichroic mirror, and emission wavelengths for this filter set are 446-512, 560 and 575$640 \mathrm{~nm}$, respectively. The exposure time is $2 \mathrm{~s}$ and AquaCosmos software (Hamamatsu) is used for the fluorescence image analysis and for correcting the data for the background detection signal. Typically, an area of $170 \times 20 \mu^{2}$ with nine consecutive magnetic chains is analyzed for the immunoassay experiments.

\section{Results and discussion}

Figure 4a shows an optical image (taken with the $100 \times$ objective) of five self-assembled magnetic chains before $\mathrm{c}-\mathrm{Ab}$ coating. During experiments, the magnetic microbeads first assemble in primary linear chains in the narrow and wide sections of the microchannel before constituting $40-\mu \mathrm{m}$-long magnetic chains that stay trapped in the $40-\mu \mathrm{m}$-large section of the microchannel. Thereby, the chains are more complex than a pearl-like chain. We 


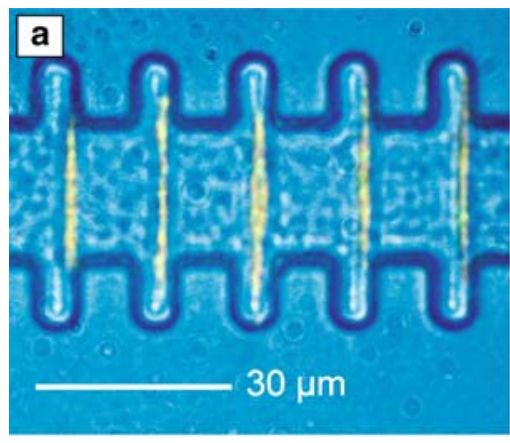

Fig. 4 a Optical image of five self-assembled magnetic chains. b Fluorescence microscopic image of 30 consecutive self-assembled magnetic microbead chains after the realization of the direct control

observe in Fig. 4a linear chains of $\sim 1.5 \mu \mathrm{m}$ diameter with local small imperfections. To estimate the number of magnetic microbeads constituting a chain, we calculate the number of magnetic microbeads initially present in one trapping unit of the microchannel (a volume of $3.2 \times 10^{-9} \mathrm{~mL}$ consisting of one large and one narrow section of the microchannel). With an initial magnetic microbead concentration of $10 \mathrm{mg} / \mathrm{mL}$, we conclude that a single magnetic chain is constituted of approximately 240 magnetic microbeads. Figure $4 \mathrm{~b}$ shows a fluorescence microscopy image of $\sim 30$ chains in the microchannel obtained after completion of the full on-chip direct control immunoassay. The two-Ab fluorescent immunocomplexes (supramolecules constituted by c-Ab and dd-Ab) are clearly located at the surface of the chains, indicating the high capture efficiency of the magnetic microbeads in our system. Figure $4 \mathrm{c}$ shows the fluorescence intensity along the microchannel axis due to the binding of the two-Ab fluorescent immunocomplexes on magnetic microbeads, as derived from images like that shown in Fig. 4b. Each peak corresponds to a single fluorescent magnetic chain. In this direct control immunoassay, all chains that are periodically trapped over the 2-mm-long microchannel (representing a total number of 120 chains) show strong fluorescence intensity. The large number of c-Ab linked to the surface of the magnetic microbeads are equivalently distributed over the 240 microbeads per chain $\times 120$ (chains) $\cong 29,000$ magnetic microbeads in the microchannel. During flowing of the $\mathrm{c}-\mathrm{Ab}$ solution, $1.22 \times 10^{8} \mathrm{c}-\mathrm{Ab}$ molecules are introduced in the microchannel. On average, 4,167 c-Ab are linked at the surface of each microbead in the microchannel, and this by only consuming $6.12 \mathrm{~nL}$ of c-Ab solution.

Figure 5a shows an optical microscopy image of the self-assembled magnetic chains in the microchannel after off-chip $\mathrm{c}-\mathrm{Ab}$ and $\mathrm{t}-\mathrm{mAb}$ incubation. The chains are accurately positioned in the geometrical traps. However, their size and homogeneity present slight variations, due to the off-chip incubation protocol. Indeed, when off-chip
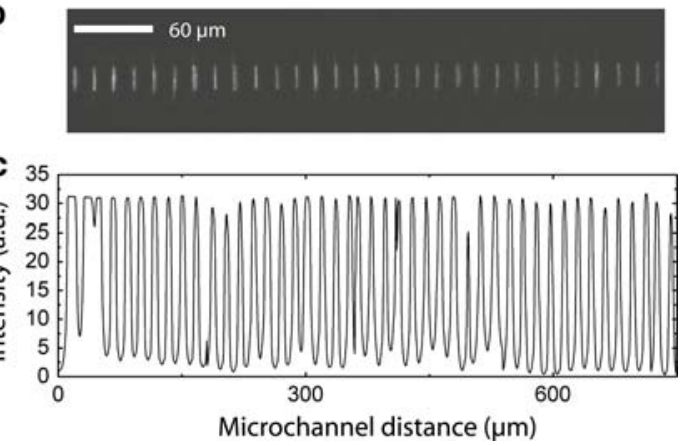

immunoassay. c Fluorescence intensity along the microchannel axis due to the formation of the two-Ab fluorescent immunocomplexes on the magnetic microbeads, as derived from images like shown in $\mathbf{b}$
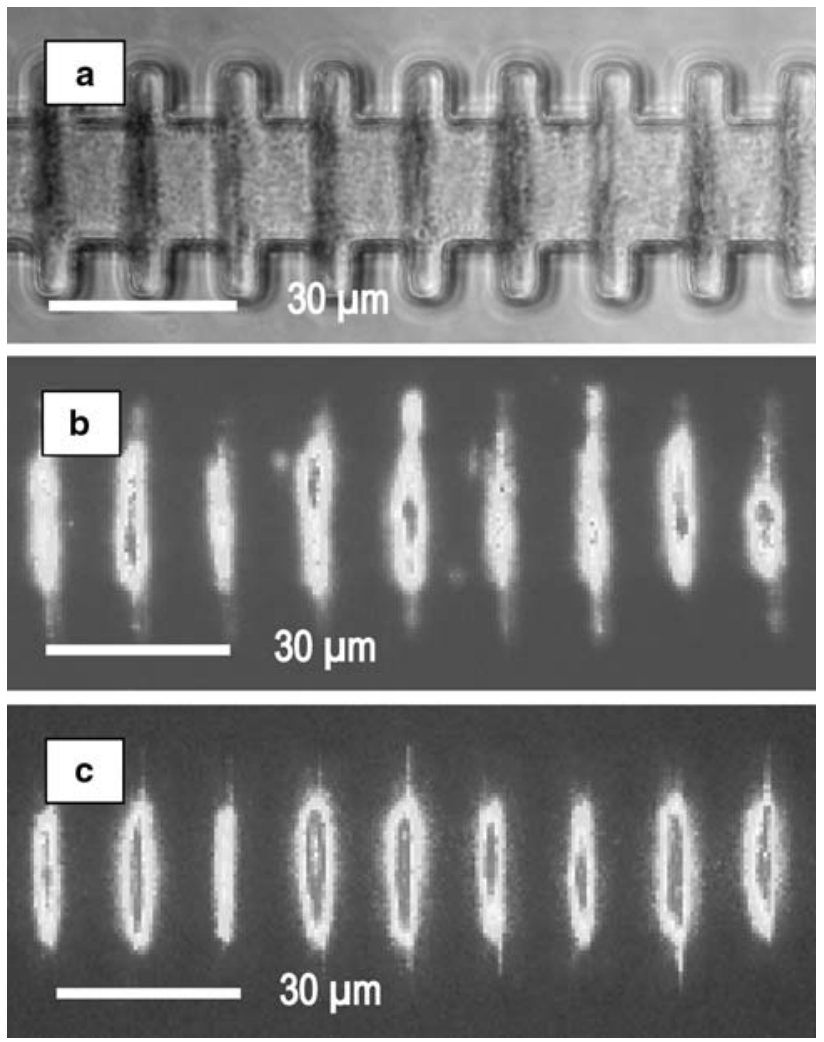

Fig. 5 a Optical image of the self-assembled magnetic chains after the off-chip c-Ab and t-mAb incubation. b Fluorescence image of the self-assembled magnetic chains in the microchannel after completion of the 'off-chip incubation-on-chip detection' sandwich immunoassay. c Fluorescence image obtained after completion of the full onchip sandwich immunoassay

incubated, the magnetic microbeads are linked by $\mathrm{c}-\mathrm{Ab}$ and form small microbead clusters. Once introduced in the microchannel, the clusters are embedded in the self-assembled magnetic chains during their formation and create chain irregularities. Figure $5 \mathrm{~b}$ shows a fluorescence image of the self-assembled magnetic chains in the microchannel after completion of the 'off-chip incubation-on-chip detection' 
sandwich immunoassay. The three-Ab fluorescent immunocomplexes (supramolecules constituted by $\mathrm{c}-\mathrm{Ab}, \mathrm{t}-\mathrm{mAb}$ and sd-Ab) are clearly located on the magnetic chains. The fluorescence signal presents variations along the chain axis due to the chain irregularities. This phenomenon is not observed in the full on-chip sandwich immunoassay (where we introduce the magnetic microbeads in the microchannel before $\mathrm{c}-\mathrm{Ab}$ coating), because the microbeads behave as independent particles which self-assemble only due to magnetic interactions when exposed to the external magnetic field. For comparison, Fig. 5c shows a fluorescence image obtained after completion of the full on-chip sandwich immunoassay. The chains are accurately positioned in the geometrical traps and are regular in size, while the fluorescent signal is homogeneous along the chain axis.

Figure 6a shows the fluorescence intensity of nine magnetic chains along a section of the microchannel, as derived from an image like shown in Fig. 5b, after completion of the 'off-chip incubation-on-chip detection' immunoassay. In a sandwich immunoassay, the limiting factor for the three-Ab immunocomplex formation is the
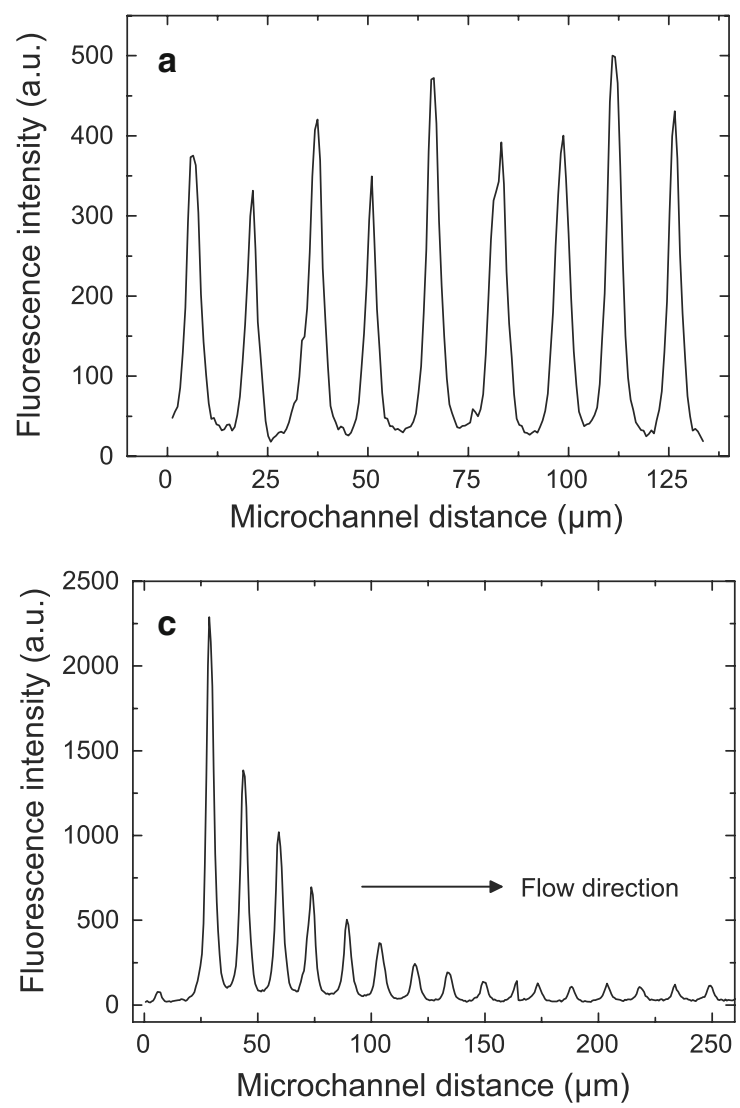

Fig. 6 a Fluorescence intensity along a section of the microchannel after completion of the 'off-chip incubation-on-chip detection' sandwich immunoassay. b Total fluorescence as a function of the t-mAb concentration for the 'off-chip incubation-on-chip detection' number of t-mAb. During the off-chip incubation, $c-A b$ and $\mathrm{t}-\mathrm{mAb}$ are homogeneously distributed over the magnetic microbead surface. The total fluorescence signal averages out chain-to-chain intensity fluctuation. The integration over all fluorescent chains on the image is proportional to the total number of $\mathrm{t}-\mathrm{mAb}$ captured on the magnetic microbeads. Figure $6 \mathrm{~b}$ shows the total integrated fluorescence as a function of the $\mathrm{t}-\mathrm{mAb}$ concentration, obtained from curves like shown in Fig. 6a. Although the average number of $\mathrm{c}-\mathrm{Ab}$ per magnetic microbead is lower than the average number of $\mathrm{t}-\mathrm{mAb}$ per bead for solution concentrations above $150 \mathrm{ng} / \mathrm{mL}$ (see Table 1), the signal increases linearly for $\mathrm{t}-\mathrm{mAb}$ concentrations between 50 and $200 \mathrm{ng} / \mathrm{nL}$. This indicates that a portion of $\mathrm{t}-\mathrm{mAb}$ molecules are probably not captured, when incubated off-chip. The 'off-chip incubation-on-chip detection' protocol is also applied to $500 \mu \mathrm{L}$ of the supernatant of a cell culture to measure the t-mAb concentration in a non-purified sample solution. The cell culture is prepared by conventional hybridoma technology (Plessers et al. 1990) in a conditioned cell culture medium to produce $\mathrm{t}-\mathrm{mAb}(5 \mathrm{D} 10$
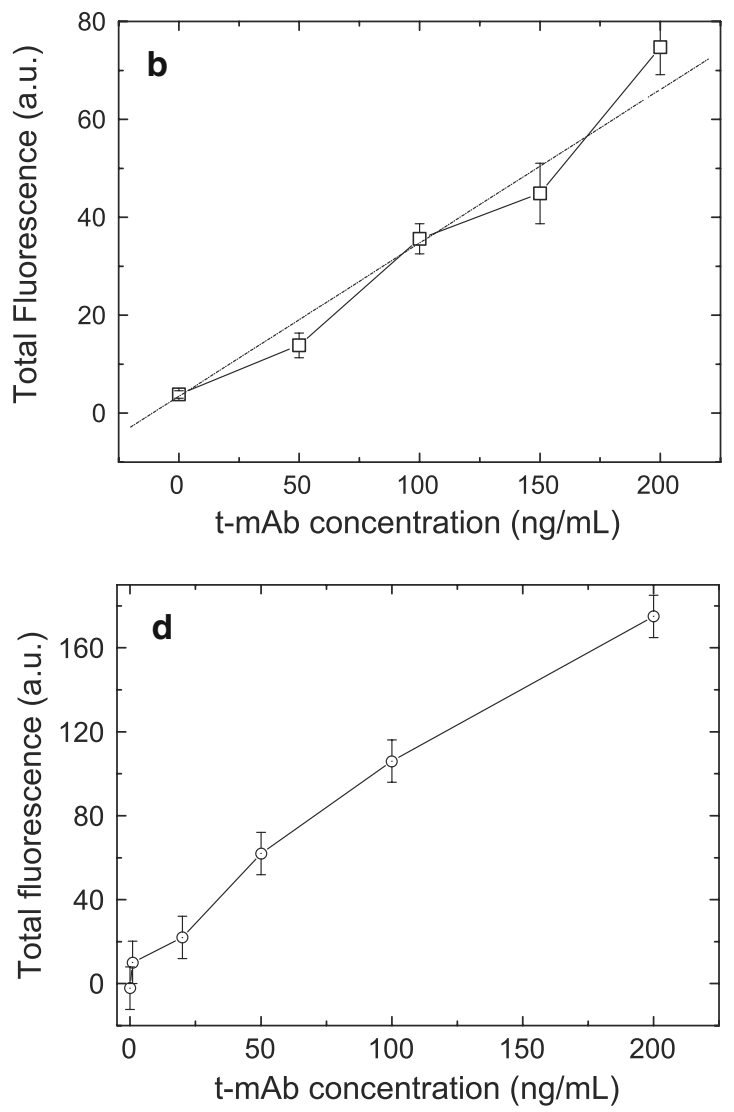

sandwich immunoassay. c Fluorescence intensity along the microchannel after the completion of the full on-chip sandwich immunoassay. d Total fluorescence as a function of the $t-m A b$ concentration for the full on-chip sandwich immunoassay 
$\mathrm{Ab}$ directed against the human breast cancer cell line MCF-7). Using the total fluorescence response curve of Fig. $6 \mathrm{~b}$, we find a concentration of $113 \pm 7 \mathrm{ng} / \mathrm{mL}$. For comparison, this concentration is measured to be $\sim 125 \mathrm{ng} /$ $\mathrm{mL}$ by a classical ELISA test (in a total assay time of $3.5 \mathrm{~h}$ ).

Figure $6 \mathrm{c}$ shows the fluorescence intensity of the first 16 chains along the microchannel after the completion of the full on-chip sandwich immunoassay, as derived from images like the one shown in Fig. 5c. The chains that are more upstream positioned in the microchannel show the highest fluorescence intensity, which is an indication of the high capture efficiency. Integrating overall fluorescent chains on the complete microchannel provides the total number of $\mathrm{t}-\mathrm{mAb}$ captured by all chains. Figure $6 \mathrm{~d}$ shows the total fluorescence intensity as a function of the $t-m A b$ concentration, obtained from curves like the one shown in Fig. 6c. As expected, the signal increases with the $t-m A b$ concentration.

It is clear from Fig. 6c that, during the full on-chip sandwich immunoassay, the magnetic chains gradually deplete the t-mAb concentration downstream of the flow. Consequently, the t-mAb molecules are not homogeneously distributed over all chains. In the absence of $\mathrm{Ab}$ saturation effects on the microbeads, the t-mAb concentration, exposing the $N$ th chain in the microchannel and proportional to its fluorescence intensity, can be written as

$C_{N}=C_{0} \times(1-P)^{N}$

where $\mathrm{C}_{0}$ is the initial concentration of $\mathrm{t}-\mathrm{mAb}$ and $P(<1)$ the capture probability of the chains. Figure 7 presents the experimental maximal fluorescent intensity extracted from

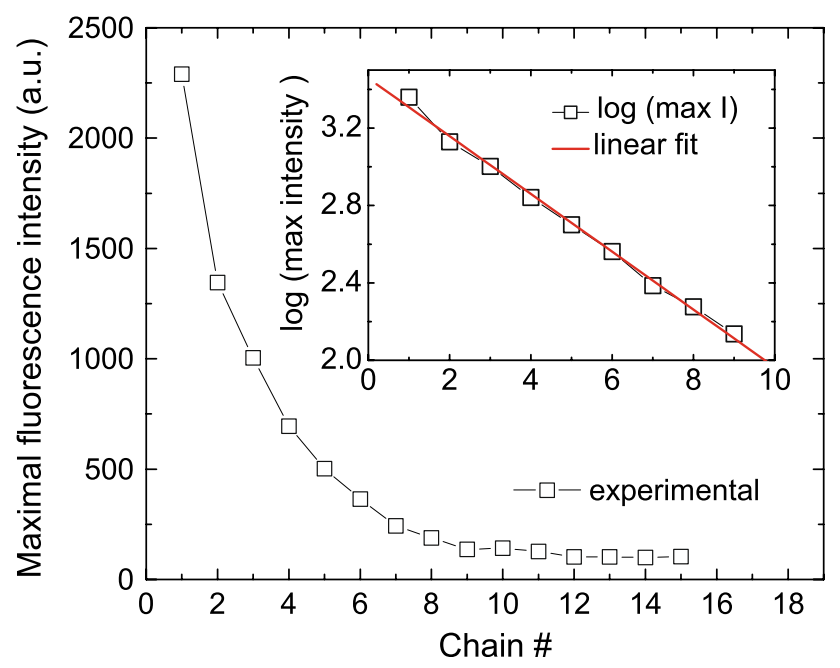

Fig. 7 Experimental maximal fluorescent intensity extracted from Fig. 6c for chains \# 1-15. The maximal fluorescent intensity follows an exponential decrease as a function of the chain number. The insert shows a single log scale plot of the maximal fluorescent intensity versus chain number for chains \# 1-9 (full on-chip sandwich immunoassay)
Fig. 6c for chains \# 1-15. As predicted by Eq. 4, the maximal fluorescent intensity follows an exponential decrease as a function of the chain number. The experimental capture probability of a chain can be extracted from experimental data by plotting on a single log scale the maximal fluorescent intensity versus chain number (see insert of Fig. 7).

$\log \left(\frac{C_{N}}{C_{0}}\right)=N \log (1-P)$

For our flow condition (flow rate of $0.034 \mathrm{~nL} / \mathrm{s}$ ), the experimental capture probability of a chain determined using Eq. 5 is found to be $P=0.29$ (i.e., $29 \%$ of all $\mathrm{t}-\mathrm{mAb}$ reaching a microchannel elementary volume, in which one chain is located, will be captured, while the rest continues to advance through the microchannel and will be captured by further chains). Both reaction kinetics and diffusion effects have an influence on the capture probability. Due to diffusion, a minimum number of chains are needed to capture all $\mathrm{t}-\mathrm{mAb}$ from the flow, even for instantaneous immunoreactions at the microbead surface. Diffusing over a distance corresponding to half of the microchannel height $h / 2=4 \mu \mathrm{m}$, with a diffusion coefficient for $\mathrm{t}-\mathrm{mAb}$ of $D=40 \times 10^{-8} \mathrm{~cm}^{2} / \mathrm{s}$, typically takes $h^{2} /(4 D) \approx 0.4 \mathrm{~s}$. Using an average flow speed of $100 \mu \mathrm{m} / \mathrm{s}$, we find that, at minimum, the flow needs to pass over a length of $40 \mu \mathrm{m}$ before all $\mathrm{t}-\mathrm{mAb}$ molecules have diffused to a magnetic chain. This corresponds to a minimum number of three chains that will show fluorescence, in qualitative agreement with the experimental results. When the flow rate decreases down to a flow velocity of $37 \mu \mathrm{m} / \mathrm{s}$, the time allowed for a $\mathrm{t}-\mathrm{mAb}$ molecule to diffuse to the chain surface will increase, and only two chains would be sufficient to capture all $t-m A b$. Decreasing further the flow rate down to a value below the typical diffusion speed $(10 \mu \mathrm{m} / \mathrm{s})$ will result in the capture of all $\mathrm{t}-\mathrm{mAb}$ by the first chain of the system.

\section{Conclusion}

We have demonstrated two protocols to perform on-chip sandwich immunoassays, using magnetic microbeads that are self-assembled in magnetic chains as reaction substrate. When incubated off-chip, t-mAb are distributed over all the magnetic microbead surfaces, and the fluorescence signal obtained reaches a similar level for all chains in the microchannel. This protocol demonstrates the extension of the use of our magnetic microbead retention device to applications involving off-chip immunoreactions with magnetic microbeads. When the $\mathrm{t}-\mathrm{mAb}$ molecules are captured from a flow in the microchannel, the magnetic chains gradually deplete the $\mathrm{t}-\mathrm{mAb}$ concentration downstream of the flow, and the t-mAb molecules are unequally 
distributed on all chains. From the spatial dependence of the fluorescence intensity, we calculate a magnetic chain capture probability of $29 \%$, primarily due to the finite diffusion times needed for the $\mathrm{t}-\mathrm{mAb}$ to reach a magnetic chain. In the 'off-chip incubation-on-chip detection' protocol, antibodies can be detected down to a concentration of $50 \mathrm{ng} / \mathrm{mL}$ in a total assay time of $120 \mathrm{~min}$, while consuming $1.5 \mathrm{~mL}$ of target antibody solution. Using the full on-chip protocol, our system is able to detect target antibodies down to a few $\mathrm{ng} / \mathrm{mL}$ in $30 \mathrm{~min}$, and using only a few tens of nanoliters of target antibody solution and reagents. Our work shows that positioning magnetic microbeads in chains across a microchannel results in a very strong particle-liquid interaction and strongly enhances the fluid perfusion through the magnetic structures. Our results suggest a high potential for immunoassays performed on-chip in our simple and versatile system, consuming lower reagent volumes and strongly reducing experimental time and costs. Moreover, we presume that the principle of magnetic chains' retention in a microfluidic flow, and incubation of the magnetic beads with analytes, either off- or on-chip, can equally well be used for other bio-analytical or catalytic applications.

\section{References}

Andersson H, van der Wijngaart W et al (2000) Micromachined flowthrough filter-chamber for chemical reactions on beads. Sens Actuators B Chem 67(1-2):203-208

Bronzeau S, Pamme N (2008) Simultaneous bioassays in a microfluidic channel on plugs of different magnetic particles. Anal Chim Acta 609(1):105-112

Choi JW, Oh KW et al (2002) An integrated microfluidic biochemical detection system for protein analysis with magnetic bead-based sampling capabilities. Lab Chip 2(1):27-30

Deng T, Prentiss M et al (2002) Fabrication of magnetic microfiltration systems using soft lithography. Appl Phys Lett 80(3):461-463

Doyle PS, Bibette J et al (2002) Self-assembled magnetic matrices for DNA separation chips. Science 295(5563):2237

Gijs MAM (2004) Magnetic bead handling on-chip: new opportunities for analytical applications. Microfluidics Nanofluidics 1(1):22-40

Hayes MA, Polson NA et al (2001) Flow-based microimmunoassay. Anal Chem 73(24):5896-5902

Helseth LE (2005) Self-assembly of colloidal pyramids in magnetic fields. Langmuir 21(16):7276-7279
Herrmann M, Veres T et al (2006) Enzymatically-generated fluorescent detection in micro-channels with internal magnetic mixing for the development of parallel microfluidic ELISA. Lab Chip 6(4):555-560

Herrmann M, Roy et al (2007) Microfluidic ELISA on non-passivated PDMS chip using magnetic bead transfer inside dual networks of channels. Lab Chip 7(11)1546-1552

Lacharme F, Vandevyver C et al (2008a) Magnetic beads retention device for on-chip sandwich immuno-assay. In: Mems 2008: 21 st IEEE international conference on micro electro mechanical systems, technical digest, pp 184-187

Lacharme F, Vandevyver C et al (2008b) Full on-chip nanoliter immunoassay by geometrical magnetic trapping of nanoparticle chains. Anal Chem 80(8):2905-2910

Lund-Olesen T, Bruus H et al (2007) Quantitative characterization of magnetic separators: comparison of systems with and without integrated microfluidic mixers. Biomed Microdevices 9(2):195205

Manz A, Graber N et al (1990) Miniaturized total chemical-analysis systems - a novel concept for chemical sensing. Sens Actuators A 10(1-6):244-248

Pavius M, Hibert C et al (2004) Profile angle control in SIO2 deep anisotropic dry etching for MEMS fabrication. In: Mems 2004: 17th IEEE international conference on micro electro mechanical systems, technical digest, pp 669-672

Peyman SA, Lies A et al (2008) Rapid on-chip multi-step (bio)chemical procedures in continuous flow-manoeuvring particles through co-laminar reagent streams. Chem Commun 10:1220 1222

Plessers L, Bosmans E et al (1990) Production and immunohistochemical reactivity of mouse antiepithelial monoclonalantibodies raised against human breast-cancer cells. Anticancer Res 10(1):271-277

Rida A, Gijs MAM (2004) Manipulation of self-assembled structures of magnetic beads for microfluidic mixing and assaying. Anal Chem 76(21):6239-6246

Rida A, Fernandez V et al (2003) Long-range transport of magnetic microbeads using simple planar coils placed in a uniform magnetostatic field. Appl Phys Lett 83(12):2396-2398

Sato K, Tokeshi M et al (2001) Determination of carcinoembryonic antigen in human sera by integrated bead bed immunoasay in a microchip for cancer diagnosis. Anal Chem 73(6):1213-1218

Shui LL, Eijkel JCT et al (2007) Multiphase flow in micro- and nanochannels. Sens Actuators B Chem 121(1):263-276

Siegel AC, Shevkoplyas SS et al (2006) Cofabrication of electromagnets and microfluldic systems in poly(dimethylsiloxane). Angew Chem Int Ed 45(41):6877-6882

Slovakova M, Minc N et al (2005) Use of self assembled magnetic beads for on-chip protein digestion. Lab Chip 5(9):935-942

Smistrup K, Kjeldsen BG et al (2005) On-chip magnetic bead microarray using hydrodynamic focusing in a passive magnetic separator. Lab Chip 5(11):1315-1319 